\title{
Stationary distribution of a stochastic SIRD epidemic model of Ebola with double saturated incidence rates and vaccination
}

Panpan Wang ${ }^{1}$ and Jianwen Jia ${ }^{1 *}$ (D)

"Correspondence:

jiajw.2008@163.com

${ }^{1}$ School of Mathematical and

Computer Science, Shanxi Normal

University, Linfen, China

\begin{abstract}
In this paper, a stochastic SIRD model of Ebola with double saturated incidence rates and vaccination is considered. Firstly, the existence and uniqueness of a global positive solution are obtained. Secondly, by constructing suitable Lyapunov functions and using Khasminskii's theory, we show that the stochastic model has a unique stationary distribution. Moreover, the extinction of the disease is also analyzed. Finally, numerical simulations are carried out to portray the analytical results.
\end{abstract}

MSC: Primary 37H10; 60H10; secondary 92C60; 92D30

Keywords: Stochastic SIRD model; Ebola virus; Extinction; Stationary distribution

\section{Introduction}

Ebola virus is a potent virus which causes Ebola hemorrhagic fever in humans and primates. It is a very rare virus. After discovering its existence in southern Sudan and the Ebola region of Congo in 1976, it attracted widespread attention in the medical community. Ebola virus has a high mortality rate between 50 percent and 90 percent. It is mainly transmitted by contacting with the blood, body fluids, and infected corpses of animals or human. For the spread of Ebola virus, on the basis of models (see e.g. Refs. [1-6]), we divide the total group into four categories: susceptible group $S$, infected group $I$, recovered group $R$, and infected corpse group $D$. The infected corpse can spread disease through contacting with the susceptible, and its infection rate is higher than that of an infected individual.

Considering the above discussion, the deterministic SIRD epidemic model is formulated as follows:

$$
\left\{\begin{array}{l}
\frac{\mathrm{d} S}{\mathrm{~d} t}=A-\frac{\beta_{1} S(t) I(t)}{1+\alpha_{1} I(t)}-\frac{\beta_{2} S(t) D(t)}{1+\alpha_{2} D(t)}-\mu S(t)-q S(t), \\
\frac{\mathrm{d} I}{\mathrm{~d} t}=\frac{\beta_{1} S(t) I(t)}{1+\alpha_{1} I(t)}-\frac{\beta_{2} S(t) D(t)}{1+\alpha_{2} D(t)}-(\rho+\mu+\delta) I(t), \\
\frac{\mathrm{d} R}{\mathrm{~d} t}=\rho I(t)+q S(t)-\mu R(t), \\
\frac{\mathrm{d} D}{\mathrm{~d} t}=(\mu+\delta) I(t)-\gamma D(t),
\end{array}\right.
$$

where $A$ is a constant input rate, $\beta_{1}$ and $\beta_{2}$ represent the transmission coefficient. Functions $\frac{\beta_{1} S(t) I(t)}{1+\alpha_{1} I(t)}$ and $\frac{\beta_{2} S(t) D(t)}{1+\alpha_{2} D(t)}$ represent two different types of saturated incidence rates. $\mu$ is

(c) The Author(s) 2019. This article is distributed under the terms of the Creative Commons Attribution 4.0 International License (http://creativecommons.org/licenses/by/4.0/), which permits unrestricted use, distribution, and reproduction in any medium, provided you give appropriate credit to the original author(s) and the source, provide a link to the Creative Commons license, and indicate if changes were made. 
the natural mortality rate of $S, I$, and $R$ compartments, $\delta$ is mortality due to diseases, $\frac{1}{\gamma}$ represents the average period of infectiousness after death in human corpses, and $\rho$ is the recovery rate. On the basis of biological significance, all parameter values are assumed to be positive, and the quantity of infected corpse is less than the quantity of infected individuals, that is, $I(t)>D(t)$, see [7].

Noticing that $R$ has no influence on the transmission dynamics, we can exclude the third equation. Therefore, we only need to study the following subsystem:

$$
\left\{\begin{array}{l}
\frac{\mathrm{d} S}{\mathrm{~d} t}=A-\frac{\beta_{1} S(t) I(t)}{1+\alpha_{1} I(t)}-\frac{\beta_{2} S(t) D(t)}{1+\alpha_{2} D(t)}-\mu S(t)-q S(t), \\
\frac{\mathrm{d} I}{\mathrm{~d} t}=\frac{\beta_{1} S(t) I(t)}{1+\alpha_{1} I(t)}-\frac{\beta_{2} S(t) D(t)}{1+\alpha_{2} D(t)}-(\rho+\mu+\delta) I(t), \\
\frac{\mathrm{d} D}{\mathrm{~d} t}=(\mu+\delta) I(t)-\gamma D(t) .
\end{array}\right.
$$

Obviously, the feasible region of system (1.2) is $\mathbf{R}_{+}^{3}$. Referring to [8], we have the basic production number of system (1.2), its expression is

$$
\Re_{0}=\frac{\beta_{1} A \gamma+\beta_{2} A(\mu+\delta)}{(\mu+q) \gamma(\rho+\mu+\delta)} .
$$

It is easy to draw two conclusions about system (1.2).

Lemma 1.1 If $\Re_{0}<1$, the disease-free equilibrium $E_{0}=\left(\frac{A}{\mu+q}, 0,0\right)$ exists, and the $E_{0}$ is globally asymptotically stable.

Lemma 1.2 If $\Re_{0}>1$, the endemic equilibrium $E_{*}=\left(S_{*}, I_{*}, D_{*}\right)$ exists, and the $E_{*}$ is globally asymptotically stable.

As mentioned above, we show that the epidemic model is depicted by deterministic model. However, epidemic models are inevitably influenced by multifarious unpredictable environmental noise types because of the actual situations. Owing to this reason, many authors have studied epidemic models with stochastic perturbations (see e.g. Refs. [918]). But as far as we know, the studies on the dynamics of the stochastic SIRD model of Ebola seem to be rare. In this paper, we assume that stochastic perturbations are of the white noise type which are directly proportional to $S(t), I(t), D(t)$, influenced on the $\dot{S}(t)$, $\dot{I}(t), \dot{D}(t)$ in system (1.2) respectively, then we build the following stochastic model:

$$
\left\{\begin{array}{l}
\mathrm{d} S(t)=\left(A-\frac{\beta_{1} S(t) I(t)}{1+\alpha_{1} I(t)}-\frac{\beta_{2} S(t) D(t)}{1+\alpha_{2} D(t)}-\mu S(t)-q S(t)\right) \mathrm{d} t+\sigma_{1} S(t) \mathrm{d} B_{1}(t), \\
\mathrm{d} I(t)=\left(\frac{\beta_{1} S(t) I(t)}{1+\alpha_{1} I(t)}+\frac{\beta_{2} S(t) D(t)}{1+\alpha_{2} D(t)}-(\rho+\mu+\delta) I(t)\right) \mathrm{d} t+\sigma_{2} I(t) \mathrm{d} B_{2}(t), \\
\mathrm{d} D(t)=((\mu+\delta) I(t)-\gamma D(t)) \mathrm{d} t+\sigma_{3} D(t) \mathrm{d} B_{3}(t),
\end{array}\right.
$$

where $\sigma_{i}(i=1,2,3)$ denotes the intensities of the white noises which satisfy nonnegativity. $B_{i}(t)(i=1,2,3)$ is a standard Brownian motion that is defined on a complete probability space $(\Omega, \mathbf{F}, \mathbf{P})$ with a filtration $\left\{\mathbf{F}_{t}\right\}_{t \in \mathbf{R}_{+}}$satisfying the usual conditions, that is, $\left\{\mathbf{F}_{t}\right\}_{t \in \mathbf{R}_{+}}$ is right continuous and $\mathbf{F}_{0}$ contains all P-null sets. Other parameters are the same as for system (1.2).

Compared with model (1.2), model (1.3) has the advantage of introducing stochastic perturbations, which makes the model closer to reality. Of course, the results of model (1.3) are more refined. 
This paper is arranged as follows. In Sect. 2, we study the existence of an ergodic stationary distribution of stochastic system (1.3). In Sect. 3, we deduce the conditions for extinction of the disease. Numerical simulations are given to support our theoretical results in Sect. 4. Finally, Sect. 5 presents some conclusions.

\section{Stationary distribution of system (1.3)}

In this section, we construct a suitable Lyapunov function to obtain the conditions of the existence of a unique ergodic stationary distribution to system (1.3). Before this, we introduce the following lemma.

Lemma 2.1 ([19]) The Markov process $X(t)$ has a unique ergodic stationary distribution $\pi(\cdot)$ if a bounded domain $D_{\varepsilon} \subset \mathbf{R}^{d}$ with regular boundary $\Gamma$ exists and

(H1) there is a constant $M>0$ satisfying $\sum_{i, j=1}^{d} a_{i j}(x) \xi_{i} \xi_{j} \geq M|\xi|^{2}, x \in D_{\varepsilon}, \xi \in \mathbf{R}^{d}$;

(H2) there exists a nonnegative $C^{2}$-function $V$ such that $L V$ is negative for any $\mathbf{R}^{d} \backslash D_{\varepsilon}$.

Then

$$
\mathbf{P}\left\{\lim _{t \rightarrow \infty} \frac{1}{T} \int_{0}^{T} f(X(t)) \mathrm{d} t=\int_{D} f(x) \pi(\mathrm{d} x)\right\}=1
$$

for all $x \in D$, where $f(\cdot)$ is an integrable function with respect to the measure $\pi$.

Theorem 2.1 For any initial value $(S(0), I(0), D(0)) \in \mathfrak{R}_{+}^{3}$, there exists a unique positive solution $(S(t), I(t), D(t))$ of system (1.3) for $t \geq 0$, and the solution will remain in $\Re_{+}^{3}$ with probability one.

The proof of this theorem is standard and we omit it.

\section{Theorem 2.2 If}

$$
\Re_{0}^{s}:=\frac{\beta_{1}}{\left(\mu+q+\frac{\sigma_{1}^{2}}{2}\right)\left(\rho+\mu+\delta+\frac{\sigma_{2}^{2}}{2}\right)}>1,
$$

then system (1.3) has a unique stationary distribution $\pi(\cdot)$, and the solution $(S(t), I(t), D(t))$ of system (1.3) is ergodic.

Proof The diffusion matrix of system (1.3) is

$$
A=\left(\begin{array}{ccc}
\sigma_{1}^{2} S^{2} & 0 & 0 \\
0 & \sigma_{2}^{2} I^{2} & 0 \\
0 & 0 & \sigma_{3}^{2} D^{2}
\end{array}\right)
$$

Let $M=\min _{(S, I, D) \in \bar{D}_{\sigma}}\left\{\sigma_{1}^{2} S^{2}, \sigma_{2}^{2} I^{2}, \sigma_{3}^{2} D^{2}\right\}$, then we can get

$$
\sum_{i, j=1}^{3} a_{i j}(x) \xi_{i} \xi_{j}=\sigma_{1}^{2} S^{2} \xi_{1}^{2}+\sigma_{2}^{2} I^{2} \xi_{2}^{2}+\sigma_{3}^{2} D^{2} \xi_{3}^{2} \geq M|\xi|^{2}, \quad(S, I, D) \in \bar{D}_{\sigma}, \xi \in \mathbf{R}_{+}^{3}
$$

Therefore, condition (H1) in Lemma 2.1 is proven.

Next, the validity of condition (H2) in Lemma 2.1 will be verified. 
We define a $\mathbf{C}^{2}$-function $\bar{V}(S, I, D): \mathbf{R}^{3} \rightarrow \mathbf{R}$ as follows:

$$
\begin{aligned}
\bar{V}(S, I, D)= & M\left(S+\left(1+\frac{\alpha_{1}}{\rho+\mu+\delta} I\right)+2 D-c_{1} \ln S-c_{2} \ln I\right) \\
& -\ln S-\ln D+\frac{1}{m+1}(S+I+D)^{m+1} \\
:= & M V_{1}+V_{2}+V_{3}+V_{4},
\end{aligned}
$$

where $M, c_{1}, c_{2}$ will be determined later. $m$ and $\delta$ are positive constants and satisfy the following inequality:

$$
\rho:=\delta-\frac{m}{2}\left(\sigma_{1}^{2} \vee \sigma_{2}^{2} \vee \sigma_{3}^{2}\right)>0, \quad \delta=\min \{\mu+q, \rho, \gamma\}
$$

Obviously,

$$
\lim _{k \rightarrow \infty,(S, I, D) \in \mathbf{R}^{3} \backslash U_{k}} \bar{V}(S, I, D)=+\infty,
$$

herein, $U_{k}=\left(\frac{1}{k}, k\right) \times\left(\frac{1}{k}, k\right) \times\left(\frac{1}{k}, k\right)$. Then, $\bar{V}(S, I, D)$ is a continuous function and has a minimum point $\left(\bar{S}_{0}, \bar{I}_{0}, \bar{D}_{0}\right)$ in the interior of $\mathbf{R}_{+}^{3}$. A nonnegative $\mathbf{C}^{2}$-function $V(S, I, D)$ : $\mathbf{R}^{3} \rightarrow \mathbf{R}$ can be defined as

$$
V(S, I, D)=\bar{V}(S, I, D)-\bar{V}\left(\bar{S}_{0}, \bar{I}_{0}, \bar{D}_{0}\right)
$$

Making use of Itô's formula, we get

$$
\begin{aligned}
L V_{1}= & A-\frac{\beta_{1} S I}{1+\alpha_{1} I}-\frac{\beta_{2} S D}{1+\alpha_{2} D}-\mu S-q S \\
& +\frac{\alpha_{1} \beta_{1} S I}{(\rho+\mu+\delta)\left(1+\alpha_{1} I\right)}+\frac{\alpha_{1} \beta_{2} S D}{(\rho+\mu+\delta)\left(1+\alpha_{2} D\right)}-\alpha_{1} I+2(\mu+\delta) I-2 \gamma D \\
& -c_{1} \frac{A}{S}+c_{1} \frac{\beta_{1} I}{1+\alpha_{1} I}+c_{1} \frac{\beta_{2} D}{1+\alpha_{2} D}+c_{1}(\mu+q)+\frac{c_{1}}{2} \sigma_{1}^{2} \\
& -c_{2} \frac{\beta_{1} S}{1+\alpha_{1} I}-c_{2} \frac{\beta_{2} S D}{I\left(1+\alpha_{2} D\right)}+c_{2}(\rho+\mu+\delta)+\frac{c_{2}}{2} \sigma_{2}^{2} \\
\leq & -\alpha_{1} I-1-c_{1} \frac{A}{S}-c_{2} \frac{\beta_{1} S}{1+\alpha_{1} I}+1+A+\frac{\alpha_{1} S}{\rho+\mu+\delta}\left[\frac{\beta_{1} I}{1+\alpha_{1} I}+\frac{\beta_{2} D}{1+\alpha_{2} D}\right] \\
& +2(\mu+\delta) I+c_{1}\left[\frac{\beta_{1} I}{1+\alpha_{1} I}+\frac{\beta_{2} D}{1+\alpha_{2} D}\right]+c_{1}(\mu+q)+\frac{c_{1}}{2} \sigma_{1}^{2} \\
& +c_{2}(\rho+\mu+\delta)+\frac{c_{2}}{2} \sigma_{2}^{2} \\
\leq & -3\left(A \beta_{1} c_{1} c_{2}\right)^{\frac{1}{3}}+c_{1}\left(\mu+q+\frac{\sigma_{1}^{2}}{2}\right)+c_{2}\left(\rho+\mu+\delta+\frac{\sigma_{2}^{2}}{2}\right)+1+A \\
& +\frac{\alpha+\mu}{\rho+\mu+\delta}\left(\frac{\beta_{1}}{\alpha_{1}}+\frac{\beta_{2}}{\alpha_{2}}\right)+2(\mu+\delta) I+c_{1}\left(\frac{\beta_{1}}{\alpha_{1}}+\frac{\beta_{2}}{\alpha_{2}}\right) .
\end{aligned}
$$


Wang and Ja Advances in Difference Equations

2019:433

Page 5 of 16

Choosing

$$
c_{1}=\frac{A}{\mu+q+\frac{\sigma_{1}^{2}}{2}}, \quad c_{2}=\frac{A}{\rho+\mu+\delta+\frac{\sigma_{2}^{2}}{2}},
$$

we have

$$
\begin{aligned}
L V_{1} \leq & -3\left[\frac{A^{3} \beta_{1}}{\left(\mu+q+\frac{\sigma_{1}^{2}}{2}\right)\left(\rho+\mu+\delta+\frac{\sigma_{2}^{2}}{2}\right)}\right]^{\frac{1}{3}}+3 A+1+\frac{\alpha_{1} S}{\rho+\mu+\delta}\left(\frac{\beta_{1}}{\alpha_{1}}+\frac{\beta_{2}}{\alpha_{2}}\right) \\
& +2(\mu+\delta) I+\frac{A}{\mu+q+\frac{\sigma_{1}^{2}}{2}}\left(\frac{\beta_{1}}{\alpha_{1}}+\frac{\beta_{2}}{\alpha_{2}}\right) \\
= & -3 A\left(\left(\Re_{0}^{S}\right)^{\frac{1}{3}}-1\right)+1+\frac{\alpha_{1} S}{\rho+\mu+\delta}\left(\frac{\beta_{1}}{\alpha_{1}}+\frac{\beta_{2}}{\alpha_{2}}\right)+2(\mu+\delta) I \\
& +\frac{A}{\mu+q+\frac{\sigma_{1}^{2}}{2}}\left(\frac{\beta_{1}}{\alpha_{1}}+\frac{\beta_{2}}{\alpha_{2}}\right) \\
:= & -\lambda+1+\frac{\alpha_{1} S}{\rho+\mu+\delta}\left(\frac{\beta_{1}}{\alpha_{1}}+\frac{\beta_{2}}{\alpha_{2}}\right)+2(\mu+\delta) I+\frac{A}{\mu+q+\frac{\sigma_{1}^{2}}{2}}\left(\frac{\beta_{1}}{\alpha_{1}}+\frac{\beta_{2}}{\alpha_{2}}\right),
\end{aligned}
$$

where $\lambda=3 A\left(\left(\Re_{0}^{S}\right)^{\frac{1}{3}}-1\right)>0$.

Applying Itô's formula, we have

$$
\begin{aligned}
L V_{2} & =-\frac{A}{S}+\frac{\beta_{1} I}{1+\alpha_{1} I}+\frac{\beta_{2} D}{1+\alpha_{2} D}+(\mu+q)+\frac{\sigma_{1}^{2}}{2} \\
& \leq-\frac{A}{S}+\frac{\beta_{1}}{\alpha_{1}}+\frac{\beta_{2}}{\alpha_{2}}+(\mu+q)+\frac{\sigma_{1}^{2}}{2}, \\
L V_{3} & =-(\mu+\delta) \frac{I}{D}+\gamma+\frac{\sigma_{3}^{2}}{2}
\end{aligned}
$$

and

$$
\begin{aligned}
L V_{4}= & (S+I+D)^{m}(A-\mu S-q S-\rho I-\gamma D) \\
& +\frac{m}{2}(S+I+D)^{m-1}\left(\sigma_{1}^{2} S^{2}+\sigma_{2}^{2} I^{2}+\sigma_{3}^{2} D^{2}\right) \\
\leq & (S+I+D)^{m}[A-\delta(S+I+D)]+\frac{m}{2}\left(\sigma_{1}^{2} \vee \sigma_{2}^{2} \vee \sigma_{3}^{2}\right)(S+I+D)^{m+1} \\
= & A(S+I+D)^{m}-\left[\delta-\frac{m}{2}\left(\sigma_{1}^{2} \vee \sigma_{2}^{2} \vee \sigma_{3}^{2}\right)\right](S+I+D)^{m+1} \\
= & F-\frac{\rho}{2}(S+I+D)^{m+1} \\
\leq & F-\frac{\rho}{2}\left(S^{m+1}+I^{m+1}+D^{m+1}\right)
\end{aligned}
$$

where

$$
F=\sup _{(S, I, D) \in \mathbf{R}_{+}^{3}}\left\{A(S+I+D)^{m}-\frac{\rho}{2}(S+I+D)^{m+1}\right\}<\infty .
$$


Therefore,

$$
\begin{aligned}
L V \leq & -M \lambda+M+\frac{\alpha_{1} M S}{\rho+\mu+\delta}\left(\frac{\beta_{1}}{\alpha_{1}}+\frac{\beta_{2}}{\alpha_{2}}\right)+2 M(\mu+\delta) I+\frac{M A}{\mu+q+\frac{\sigma_{1}^{2}}{2}}\left(\frac{\beta_{1}}{\alpha_{1}}+\frac{\beta_{2}}{\alpha_{2}}\right) \\
& -\frac{A}{S}+\frac{\beta_{1}}{\alpha_{1}}+\frac{\beta_{2}}{\alpha_{2}}+(\mu+q+\gamma)-(\mu+\delta) \frac{I}{D}+\frac{\sigma_{1}^{2}+\sigma_{3}^{2}}{2} \\
& +F-\frac{\rho}{2}\left(S^{m+1}+I^{m+1}+D^{m+1}\right) .
\end{aligned}
$$

We can choose a positive constant $M$ such that

$$
-M \lambda+E \leq-2,
$$

herein,

$$
\begin{aligned}
E= & \sup _{(S, I, D) \in \mathbf{R}_{+}^{3}}\left\{M+\frac{\alpha_{1} M S}{\rho+\mu+\delta}\left(\frac{\beta_{1}}{\alpha_{1}}+\frac{\beta_{2}}{\alpha_{2}}\right)+\frac{M A}{\mu+q+\frac{\sigma_{1}^{2}}{2}}\left(\frac{\beta_{1}}{\alpha_{1}}+\frac{\beta_{2}}{\alpha_{2}}\right)\right. \\
& \left.+\left(\frac{\beta_{1}}{\alpha_{1}}+\frac{\beta_{2}}{\alpha_{2}}\right)+(\mu+q+\gamma)+\frac{\sigma_{1}^{2}+\sigma_{3}^{2}}{2}+F-\frac{\rho}{2}\left(S^{m+1}+I^{m+1}+D^{m+1}\right)\right\} .
\end{aligned}
$$

Now, we construct the following bounded closed set:

$$
D_{\varepsilon}=\left\{(S, I, D) \in \mathbf{R}_{+}^{3}: \varepsilon \leq S \leq \frac{1}{\varepsilon}, \varepsilon \leq I \leq \frac{1}{\varepsilon}, \varepsilon^{2} \leq D \leq \frac{1}{\varepsilon^{2}}\right\}
$$

where $\varepsilon>0$ is a sufficiently small constant. In the set $\mathbf{R}_{+}^{3} \backslash D_{\varepsilon}$, we can choose $\varepsilon$ sufficiently small and the following conditions hold:

$$
\begin{aligned}
& -\frac{A}{\varepsilon}+G \leq-1, \\
& -M \lambda+2 M(\mu+\delta) \varepsilon+E \leq-1, \\
& -\frac{\mu+\delta}{\varepsilon}+G \leq-1, \\
& -\frac{\rho}{4} \frac{1}{\varepsilon^{m+1}}+H \leq-1, \\
& -\frac{\rho}{4} \frac{1}{\varepsilon^{m+1}}+J \leq-1, \\
& -\frac{\rho}{4} \frac{1}{\varepsilon^{2 m+2}}+N \leq-1,
\end{aligned}
$$

where $G, H, J, N$ will be determined later. Hence,

$$
\mathbf{R}_{+}^{3} \backslash D_{\varepsilon}=D_{1}^{c} \cup D_{2}^{c} \cup D_{3}^{c} \cup D_{4}^{c} \cup D_{5}^{c} \cup D_{6}^{c},
$$

with

$$
D_{1}^{c}=\{(S, I, D) \in \Omega, 0<S<\varepsilon\}, \quad D_{2}^{c}=\{(S, I, D) \in \Omega, 0<I<\varepsilon\},
$$




$$
\begin{aligned}
& D_{3}^{c}=\{(S, I, D) \in \Omega, 0<D<\varepsilon, I \geq \varepsilon\}, \quad D_{4}^{c}=\left\{(S, I, D) \in \Omega, S>\frac{1}{\varepsilon}\right\}, \\
& D_{5}^{c}=\left\{(S, I, D) \in \Omega, I \geq \frac{1}{\varepsilon}\right\}, \quad D_{6}^{c}=\left\{(S, I, D) \in \Omega, D>\frac{1}{\varepsilon^{2}}\right\},
\end{aligned}
$$

then we will prove that $L V(S, I, D) \leq-1$ on $\mathbf{R}_{+}^{3} \backslash D_{\varepsilon}$.

Case 1. If $(S, I, D) \in D_{1}^{c}$, we achieve that

$$
\begin{aligned}
L V \leq & -M \lambda+M+\frac{\alpha_{1} M S}{\rho+\mu+\delta}\left(\frac{\beta_{1}}{\alpha_{1}}+\frac{\beta_{2}}{\alpha_{2}}\right)+2 M(\mu+\delta) I+\frac{M A}{\mu+q+\frac{\sigma_{1}^{2}}{2}}\left(\frac{\beta_{1}}{\alpha_{1}}+\frac{\beta_{2}}{\alpha_{2}}\right) \\
& -\frac{A}{S}+\left(\frac{\beta_{1}}{\alpha_{1}}+\frac{\beta_{2}}{\alpha_{2}}\right)+(\mu+q+\gamma)+\frac{\sigma_{1}^{2}+\sigma_{3}^{2}}{2}+F \\
& -\frac{\rho}{2}\left(S^{m+1}+I^{m+1}+D^{m+1}\right) \\
\leq & -\frac{A}{S}+G \\
\leq & -\frac{A}{\varepsilon}+G
\end{aligned}
$$

where

$$
\begin{aligned}
G= & \sup _{(S, I, D) \in \mathbf{R}_{+}^{3}}\left\{M+\frac{\alpha_{1} M S}{\rho+\mu+\delta}\left(\frac{\beta_{1}}{\alpha_{1}}+\frac{\beta_{2}}{\alpha_{2}}\right)+2 M(\mu+\delta) I+\frac{M A}{\mu+q+\frac{\sigma_{1}^{2}}{2}}\left(\frac{\beta_{1}}{\alpha_{1}}+\frac{\beta_{2}}{\alpha_{2}}\right)\right. \\
& \left.+\left(\frac{\beta_{1}}{\alpha_{1}}+\frac{\beta_{2}}{\alpha_{2}}\right)+(\mu+q+\gamma)+\frac{\sigma_{1}^{2}+\sigma_{3}^{2}}{2}+F-\frac{\rho}{2}\left(S^{m+1}+I^{m+1}+D^{m+1}\right)\right\} .
\end{aligned}
$$

Making use of (2.4), we have that $L V \leq-1$ for all $(S, I, D) \in D_{1}^{c}$.

Case 2. If $(S, I, D) \in D_{2}^{c}$, we obtain that

$$
\begin{aligned}
L V \leq & -M \lambda+M+\frac{\alpha_{1} M S}{\rho+\mu+\delta}\left(\frac{\beta_{1}}{\alpha_{1}}+\frac{\beta_{2}}{\alpha_{2}}\right)+2 M(\mu+\delta) I+\frac{M A}{\mu+q+\frac{\sigma_{1}^{2}}{2}}\left(\frac{\beta_{1}}{\alpha_{1}}+\frac{\beta_{2}}{\alpha_{2}}\right) \\
& -\frac{A}{S}+\left(\frac{\beta_{1}}{\alpha_{1} I}+\frac{\beta_{2}}{\alpha_{2}}\right)+(\mu+q+\gamma)+\frac{\sigma_{1}^{2}+\sigma_{3}^{2}}{2}+F \\
& -\frac{\rho}{2}\left(S^{m+1}+I^{m+1}+D^{m+1}\right) \\
\leq & -M \lambda+2 M(\mu+\delta) I+E \\
\leq & -M \lambda+2 M(\mu+\delta) \varepsilon+E .
\end{aligned}
$$

By inequality (2.5), we can achieve that $L V \leq-1$ for all $(S, I, D) \in D_{2}^{c}$.

Case 3. If $(S, I, D) \in D_{3}^{c}$, we get that

$$
\begin{aligned}
L V \leq & -(\mu+\delta) \frac{I}{D}+M+\frac{\alpha_{1} M S}{\rho+\mu+\delta}\left(\frac{\beta_{1}}{\alpha_{1}}+\frac{\beta_{2}}{\alpha_{2}}\right)+2 M(\mu+\delta) I+\frac{M A}{\mu+q+\frac{\sigma_{1}^{2}}{2}}\left(\frac{\beta_{1}}{\alpha_{1}}+\frac{\beta_{2}}{\alpha_{2}}\right) \\
& +\left(\frac{\beta_{1}}{\alpha_{1} I}+\frac{\beta_{2}}{\alpha_{2}}\right)+(\mu+q+\gamma)+\frac{\sigma_{1}^{2}+\sigma_{3}^{2}}{2}+F-\frac{\rho}{2}\left(S^{m+1}+I^{m+1}+D^{m+1}\right) \\
\leq & -\frac{\mu+\delta}{\varepsilon}+G .
\end{aligned}
$$


Wang and Ja Advances in Difference Equations

(2019) 2019:433

Page 8 of 16

Applying (2.6), we have that $L V \leq-1$ for all $(S, I, D) \in D_{3}^{c}$.

Case 4. If $(S, I, D) \in D_{4}^{c}$, we have that

$$
\begin{aligned}
L V \leq & -\frac{1}{4}\left[\delta-\frac{m}{2}\left(\sigma_{1}^{2} \vee \sigma_{2}^{2} \vee \sigma_{3}^{2}\right)\right] S^{m+1}-\frac{1}{4}\left[\delta-\frac{m}{2}\left(\sigma_{1}^{2} \vee \sigma_{2}^{2} \vee \sigma_{3}^{2}\right)\right] S^{m+1} \\
& -\frac{1}{2}\left[\delta-\frac{m}{2}\left(\sigma_{1}^{2} \vee \sigma_{2}^{2} \vee \sigma_{3}^{2}\right)\right]\left(I^{m+1}+D^{m+1}\right)+M+\frac{\alpha_{1} M S}{\rho+\mu+\delta}\left(\frac{\beta_{1}}{\alpha_{1}}+\frac{\beta_{2}}{\alpha_{2}}\right) \\
& +2 M(\mu+\delta) I+\frac{M A}{\mu+q+\frac{\sigma_{1}^{2}}{2}}\left(\frac{\beta_{1}}{\alpha_{1}}+\frac{\beta_{2}}{\alpha_{2}}\right)+\left(\frac{\beta_{1}}{\alpha_{1} I}+\frac{\beta_{2}}{\alpha_{2}}\right)+(\mu+q+\gamma) \\
& +\frac{\sigma_{1}^{2}+\sigma_{3}^{2}}{2}+F \\
\leq & -\frac{\rho}{4} S^{m+1}+H \\
\leq & -\frac{\rho}{4} \frac{1}{\varepsilon^{m+1}}+H
\end{aligned}
$$

where

$$
\begin{aligned}
H= & \sup _{(S, I, D) \in \mathbf{R}_{+}^{3}}\left\{-\frac{1}{4}\left[\delta-\frac{m}{2}\left(\sigma_{1}^{2} \vee \sigma_{2}^{2} \vee \sigma_{3}^{2}\right)\right] S^{m+1}\right. \\
& -\frac{1}{2}\left[\delta-\frac{m}{2}\left(\sigma_{1}^{2} \vee \sigma_{2}^{2} \vee \sigma_{3}^{2}\right)\right]\left(I^{m+1}+D^{m+1}\right) \\
& +M+\frac{\alpha_{1} M S}{\rho+\mu+\delta}\left(\frac{\beta_{1}}{\alpha_{1}}+\frac{\beta_{2}}{\alpha_{2}}\right)+2 M(\mu+\delta) I+\frac{M A}{\mu+q+\frac{\sigma_{1}^{2}}{2}}\left(\frac{\beta_{1}}{\alpha_{1}}+\frac{\beta_{2}}{\alpha_{2}}\right) \\
& \left.+\left(\frac{\beta_{1}}{\alpha_{1} I}+\frac{\beta_{2}}{\alpha_{2}}\right)+(\mu+q+\gamma)+\frac{\sigma_{1}^{2}+\sigma_{3}^{2}}{2}+F\right\} .
\end{aligned}
$$

Combining with (2.7), it can be obtained that $L V \leq-1$ for all $(S, I, D) \in D_{4}^{c}$.

Case 5. If $(S, I, D) \in D_{5}^{c}$, we can see that

$$
\begin{aligned}
L V \leq & -\frac{1}{4}\left[\delta-\frac{m}{2}\left(\sigma_{1}^{2} \vee \sigma_{2}^{2} \vee \sigma_{3}^{2}\right)\right] I^{m+1}-\frac{1}{4}\left[\delta-\frac{m}{2}\left(\sigma_{1}^{2} \vee \sigma_{2}^{2} \vee \sigma_{3}^{2}\right)\right] I^{m+1} \\
& -\frac{1}{2}\left[\delta-\frac{m}{2}\left(\sigma_{1}^{2} \vee \sigma_{2}^{2} \vee \sigma_{3}^{2}\right)\right]\left(S^{m+1}+D^{m+1}\right)+M+\frac{\alpha_{1} M S}{\rho+\mu+\delta}\left(\frac{\beta_{1}}{\alpha_{1}}+\frac{\beta_{2}}{\alpha_{2}}\right) \\
& +2 M(\mu+\delta) I+\frac{M A}{\mu+q+\frac{\sigma_{1}^{2}}{2}}\left(\frac{\beta_{1}}{\alpha_{1}}+\frac{\beta_{2}}{\alpha_{2}}\right)+\left(\frac{\beta_{1}}{\alpha_{1} I}+\frac{\beta_{2}}{\alpha_{2}}\right)+(\mu+q+\gamma) \\
& +\frac{\sigma_{1}^{2}+\sigma_{3}^{2}}{2}+F \\
\leq & -\frac{\rho}{4} I^{m+1}+J \\
\leq & -\frac{\rho}{4} \frac{1}{\varepsilon^{m+1}}+J
\end{aligned}
$$

where

$$
J=\sup _{(S, I, D) \in \mathbf{R}_{+}^{3}}\left\{-\frac{1}{4}\left[\delta-\frac{m}{2}\left(\sigma_{1}^{2} \vee \sigma_{2}^{2} \vee \sigma_{3}^{2}\right)\right] I^{m+1}\right.
$$




$$
\begin{aligned}
& -\frac{1}{2}\left[\delta-\frac{m}{2}\left(\sigma_{1}^{2} \vee \sigma_{2}^{2} \vee \sigma_{3}^{2}\right)\right]\left(S^{m+1}+D^{m+1}\right) \\
& +M+\frac{\alpha_{1} M S}{\rho+\mu+\delta}\left(\frac{\beta_{1}}{\alpha_{1}}+\frac{\beta_{2}}{\alpha_{2}}\right)+2 M(\mu+\delta) I+\frac{M A}{\mu+q+\frac{\sigma_{1}^{2}}{2}}\left(\frac{\beta_{1}}{\alpha_{1}}+\frac{\beta_{2}}{\alpha_{2}}\right) \\
& \left.+\left(\frac{\beta_{1}}{\alpha_{1} I}+\frac{\beta_{2}}{\alpha_{2}}\right)+(\mu+q+\gamma)+\frac{\sigma_{1}^{2}+\sigma_{3}^{2}}{2}+F\right\}
\end{aligned}
$$

According to inequality (2.8), it can be achieved that $L V \leq-1$ for all $(S, I, D) \in D_{5}^{c}$.

Case 6. If $(S, I, D) \in D_{6}^{c}$, we can see that

$$
\begin{aligned}
L V \leq & -\frac{1}{4}\left[\delta-\frac{m}{2}\left(\sigma_{1}^{2} \vee \sigma_{2}^{2} \vee \sigma_{3}^{2}\right)\right] D^{m+1}-\frac{1}{4}\left[\delta-\frac{m}{2}\left(\sigma_{1}^{2} \vee \sigma_{2}^{2} \vee \sigma_{3}^{2}\right)\right] D^{m+1} \\
& -\frac{1}{2}\left[\delta-\frac{m}{2}\left(\sigma_{1}^{2} \vee \sigma_{2}^{2} \vee \sigma_{3}^{2}\right)\right]\left(S^{m+1}+I^{m+1}\right)+M+\frac{\alpha_{1} M S}{\rho+\mu+\delta}\left(\frac{\beta_{1}}{\alpha_{1}}+\frac{\beta_{2}}{\alpha_{2}}\right) \\
& +2 M(\mu+\delta) I+\frac{M A}{\mu+q+\frac{\sigma_{1}^{2}}{2}}\left(\frac{\beta_{1}}{\alpha_{1}}+\frac{\beta_{2}}{\alpha_{2}}\right)+\left(\frac{\beta_{1}}{\alpha_{1} I}+\frac{\beta_{2}}{\alpha_{2}}\right)+(\mu+q+\gamma) \\
& +\frac{\sigma_{1}^{2}+\sigma_{3}^{2}}{2}+F \\
\leq & -\frac{\rho}{4} D^{m+1}+N \\
\leq & -\frac{\rho}{4} \frac{1}{\varepsilon^{2 m+2}}+N
\end{aligned}
$$

where

$$
\begin{aligned}
N= & \sup _{(S, I, D) \in \mathbf{R}_{+}^{3}}\left\{-\frac{1}{4}\left[\delta-\frac{m}{2}\left(\sigma_{1}^{2} \vee \sigma_{2}^{2} \vee \sigma_{3}^{2}\right)\right] D^{m+1}\right. \\
& -\frac{1}{2}\left[\delta-\frac{m}{2}\left(\sigma_{1}^{2} \vee \sigma_{2}^{2} \vee \sigma_{3}^{2}\right)\right]\left(S^{m+1}+I^{m+1}\right) \\
& +M+\frac{\alpha_{1} M S}{\rho+\mu+\delta}\left(\frac{\beta_{1}}{\alpha_{1}}+\frac{\beta_{2}}{\alpha_{2}}\right)+2 M(\mu+\delta) I+\frac{M A}{\mu+q+\frac{\sigma_{1}^{2}}{2}}\left(\frac{\beta_{1}}{\alpha_{1}}+\frac{\beta_{2}}{\alpha_{2}}\right) \\
& \left.+\left(\frac{\beta_{1}}{\alpha_{1} I}+\frac{\beta_{2}}{\alpha_{2}}\right)+(\mu+q+\gamma)+\frac{\sigma_{1}^{2}+\sigma_{3}^{2}}{2}+F\right\} .
\end{aligned}
$$

By inequality (2.9), it can be seen that $L V \leq-1$ for all $(S, I, D) \in D_{6}^{c}$.

Thus condition (H2) of Lemma 2.1 holds. By Lemma 2.1, we know that the solution of system (1.3) is ergodic and system (1.3) has a unique stationary distribution $\pi(\cdot)$.

This completes the proof.

Remark 1 Here, we construct a $\mathbf{C}^{2}$-function, and then we are going to do a step by step calculation of the function, to make the calculation process more logical. Then, the discussion is divided into six cases. Finally, the ideal result is obtained.

\section{Extinction}

In this section, we give the sufficient conditions that lead to the extinction of the disease. For convenience, we introduce the following notation and lemma. 
If $f(t)$ is an integral function on $[0, \infty)$, define $\langle f(t)\rangle=\frac{1}{t} \int_{0}^{t} f(\tau) \mathrm{d} \tau$.

Lemma 3.1 Let $(S(t), I(t), D(t))$ be the solution of system (1.3) with the initial value $(S(0), I(0), D(0)) \in \mathbf{R}_{+}^{3}$. Then $S(t)+I(t) \leq \frac{A}{\mu}, D(t) \leq \frac{A(\mu+\delta)}{\gamma \mu}$.

Proof Summing the first two equations of system (1.3), we can get

$$
\frac{\mathrm{d}(S(t)+I(t))}{\mathrm{d} t} \leq A-\mu(S(t)+I(t))
$$

so we have

$$
\limsup _{t \rightarrow \infty}(S(t)+I(t)) \leq \frac{A}{\mu} .
$$

In the same way, we have

$$
\limsup _{t \rightarrow \infty} D(t) \leq \frac{A(\mu+\delta)}{\gamma \mu} .
$$

Therefore, the positive invariant set of system (1.3) is

$$
\Omega=\left\{(S, I, D) \in \mathbf{R}_{+}^{3}: 0 \leq S, I \leq \frac{A}{\mu}, \quad 0 \leq D \leq \frac{A(\mu+\delta)}{\gamma \mu}\right\} .
$$

This completes the proof of Lemma 3.1.

Lemma 3.2 Let $(S(t), I(t), D(t))$ be the solution of system (1.3) with the initial value $(S(0), I(0), D(0)) \in \Omega$. Then

$$
\lim _{t \rightarrow \infty} \frac{\int_{0}^{t} \sigma_{1} S(\tau) \mathrm{d} B_{1}(\tau)}{t}=0, \quad \lim _{t \rightarrow \infty} \frac{\int_{0}^{t} \sigma_{2} I(\tau) \mathrm{d} B_{2}(\tau)}{t}=0 .
$$

Proof Let $M_{1}(t)=\int_{0}^{t} \sigma_{1} S(\tau) \mathrm{d} B_{1}(\tau), M_{1}(t)$ is an integral and corresponds to the Brownian motion and it is local continuous martingale. Also, if we replace the upper bound with $t=0$ in $M_{1}(t)$, then we have $M(0)=0$. Next, we can discover the quadratic variation and get the following limits:

$$
\limsup _{t \rightarrow \infty} \frac{\left\langle M_{1}, M_{1}\right\rangle_{t}}{t} \leq \frac{\sigma_{1}^{2} A^{2}}{\mu^{2}}<\infty
$$

Applying the strong law of large numbers [9], we can deduce that

$$
\lim _{t \rightarrow \infty} \frac{M_{1}(t)}{t}=0 \quad \text { a.s. }
$$

Let $M_{2}(t)=\int_{0}^{t} \sigma_{2} I(\tau) \mathrm{d} B_{2}(\tau)$. Similarly, we can get

$$
\limsup _{t \rightarrow \infty} \frac{\left\langle M_{2}, M_{2}\right\rangle_{t}}{t} \leq \frac{\sigma_{2}^{2} A^{2}}{\mu^{2}}<\infty
$$


then

$$
\lim _{t \rightarrow \infty} \frac{M_{2}(t)}{t}=0 \quad \text { a.s. }
$$

This completes the proof of Lemma 3.2.

Theorem 3.1 Let $(S(t), I(t), D(t))$ be the solution of system (1.3) with the initial value $(S(0), I(0), D(0)) \in \mathfrak{R}_{+}^{3} . I f$

$$
\tilde{\Re}_{0}^{s}:=\frac{\left(\beta_{1}+\beta_{2}\right) A}{(\mu+q)\left(\rho+\mu+\delta+\frac{\sigma_{2}^{2}}{2}\right)}<1,
$$

then

$$
\limsup _{t \rightarrow \infty} \frac{\ln I(t)}{t} \leq\left(\rho+\mu+\delta+\frac{\sigma_{2}^{2}}{2}\right)\left(\tilde{\Re}_{0}^{s}-1\right)<0 \quad \text { a.s. },
$$

that is,

$$
\lim _{t \rightarrow \infty} I(t)=0 \quad \text { a.s. }
$$

which means the disease dies out with probability one.

Moreover,

$$
\lim _{t \rightarrow \infty} S(t)=\frac{A}{\mu+q} \quad \text { a.s., } \quad \lim _{t \rightarrow \infty} D(t)=0 \quad \text { a.s. }
$$

Proof Integrating system (1.3), we get

$$
\begin{aligned}
\frac{S(t)-S(0)}{t}+\frac{I(t)-I(0)}{t}= & A-(\mu+q)\langle S(t)\rangle-(\rho+\mu+\delta)\langle I(t)\rangle \\
& +\frac{\sigma_{1}}{t} \int_{0}^{t} \sigma_{1} S(\tau) \mathrm{d} B_{1}(\tau)+\frac{\sigma_{2}}{t} \int_{0}^{t} \sigma_{2} I(\tau) \mathrm{d} B_{2}(\tau),
\end{aligned}
$$

which means

$$
\langle S(t)\rangle=\frac{A}{\mu+q}-\frac{\rho+\mu+\delta}{\mu+q}\langle I(t)\rangle+\Phi(t),
$$

where

$$
\Phi(t)=\frac{1}{\mu+q}\left[\frac{\sigma_{1}}{t} \int_{0}^{t} S(\tau) \mathrm{d} B_{1}(\tau)+\frac{\sigma_{2}}{t} \int_{0}^{t} I(\tau) \mathrm{d} B_{2}(\tau)-\frac{S(t)-S(0)}{t}-\frac{I(t)-I(0)}{t}\right] .
$$

By Lemma 3.1 and Lemma 3.2 we can obtain

$$
\lim _{t \rightarrow \infty} \Phi(t)=0 \quad \text { a.s. }
$$

Applying Itô’s formula to system (1.3), we get that

$$
\mathrm{d} \ln I(t)=\frac{\beta_{1} S(t)}{1+\alpha_{1} I(t)}+\frac{\beta_{2} S(t) D(t)}{I(t)\left(1+\alpha_{2} D(t)\right)}-(\rho+\mu+\delta)-\frac{\sigma_{2}^{2}}{2}+\sigma_{2} \mathrm{~d} B_{2}(t) .
$$


Integrating both sides of (3.4) from 0 to $t$ and division by $t$ lead to the following equation:

$$
\begin{aligned}
\frac{\ln I(t)}{t}= & \beta_{1}\left\langle\frac{S(t)}{1+\alpha_{1} I(t)}\right\rangle+\beta_{2}\left\langle\frac{S(t) D(t)}{I(t)\left(1+\alpha_{2} D(t)\right)}\right\rangle-(\rho+\mu+\delta)-\frac{\sigma_{2}^{2}}{2}+\frac{\sigma_{2} B_{2}(t)}{t} \\
\leq & \beta_{1}\langle S(t)\rangle+\beta_{2}\langle S(t)\rangle-(\rho+\mu+\delta)-\frac{\sigma_{2}^{2}}{2}+\frac{\sigma_{2} B_{2}(t)}{t} \\
= & \left(\beta_{1}+\beta_{2}\right)\left[\frac{A}{\mu+q}-\frac{\rho+\mu+\delta}{\mu+q}\langle I(t)\rangle+\Phi(t)\right]-(\rho+\mu+\delta)-\frac{\sigma_{2}^{2}}{2}+\frac{\sigma_{2} B_{2}(t)}{t} \\
= & \frac{\left(\beta_{1}+\beta_{2}\right) A}{\mu+q}-\frac{\left(\beta_{1}+\beta_{2}\right)(\rho+\mu+\delta)}{\mu+q}\langle I(t)\rangle+\left(\beta_{1}+\beta_{2}\right) \Phi(t) \\
& -(\rho+\mu+\delta)-\frac{\sigma_{2}^{2}}{2}+\frac{\sigma_{2} B_{2}(t)}{t} \\
= & \left(\rho+\mu+\delta+\frac{\sigma_{2}^{2}}{2}\right)\left(\tilde{\Re}_{0}^{s}-1\right)-\frac{\left(\beta_{1}+\beta_{2}\right)(\rho+\mu+\delta)}{\mu+q}\langle I(t)\rangle \\
& +\left(\beta_{1}+\beta_{2}\right) \Phi(t)+\frac{\sigma_{2} B_{2}(t)}{t} \\
\leq & \left(\rho+\mu+\delta+\frac{\sigma_{2}^{2}}{2}\right)\left(\tilde{\Re}_{0}^{s}-1\right)+\left(\beta_{1}+\beta_{2}\right) \Phi(t)+\frac{\sigma_{2} B_{2}(t)}{t} .
\end{aligned}
$$

From strong law of large numbers [9], we have

$$
\lim _{t \rightarrow \infty} \frac{\sigma_{2} B_{2}(t)}{t}=0 \quad \text { a.s. }
$$

Combining (3.3) and (3.6), then taking the limit superior of both sides of (3.5), we have

$$
\limsup _{t \rightarrow \infty} \frac{\ln I(t)}{t} \leq\left(\rho+\mu+\delta+\frac{\sigma_{2}^{2}}{2}\right)\left(\tilde{\Re}_{0}^{s}-1\right) \quad \text { a.s. }
$$

This implies that if $\tilde{\mathfrak{R}}_{0}^{s}<1$, then

$$
\lim _{t \rightarrow \infty} I(t)=0 \quad \text { a.s. }
$$

According to system (1.3), the third equation with limiting system becomes

$$
\mathrm{d} D(t)=-\gamma D(t) \mathrm{d} t
$$

thus, we obtain

$$
\lim _{t \rightarrow \infty} D(t)=0 \quad \text { a.s. }
$$

Similarly, according to the first equation of (1.3), we have

$$
\lim _{t \rightarrow \infty} S(t)=\frac{A}{\mu+q} \quad \text { a.s. }
$$

This completes the proof of Theorem 3.1. 

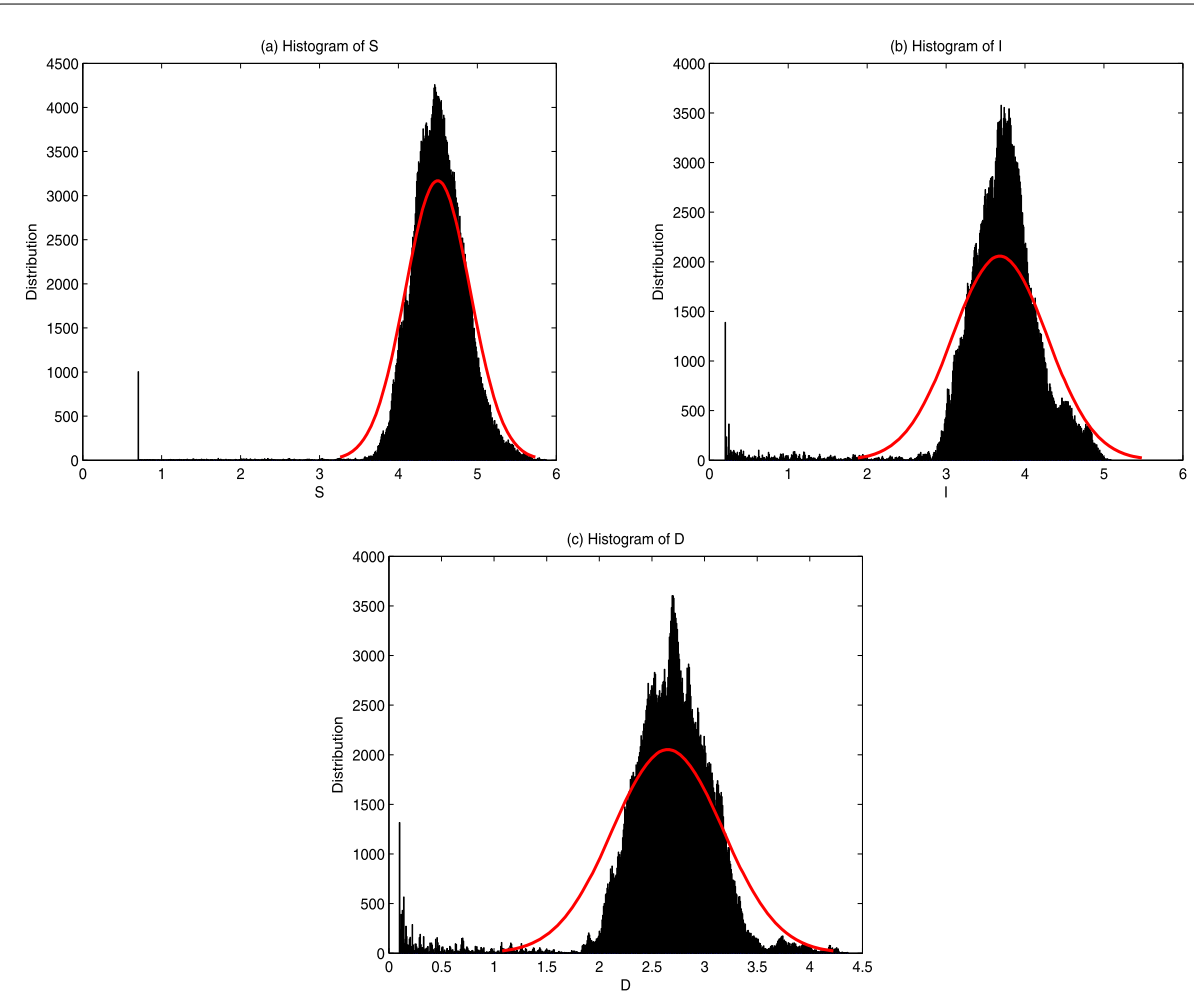

Figure 1 Distributions of $S(t), I(t)$, and $D(t)$ of system (1.3) with $\sigma_{1}=\sigma_{2}=\sigma_{3}=0.1$

Remark 2 Theorem 3.1 shows that the disease will become extinct if $\tilde{\Re}_{0}^{s}<1$. Note that $\tilde{\Re}_{0}^{s}<1$ independent of $\sigma_{1}$ and $\sigma_{3}$, that is to say, when $\tilde{\Re}_{0}^{s}<1$, even if the white noises $\sigma_{1}$ and $\sigma_{3}$ are zero, the disease is still extinct.

\section{Numerical simulations}

In this section, we use the Milstein method [20] and numerical simulations to verify the conclusions in this paper. The discrimination equations of system (1.3) are presented as follows:

$$
\begin{gathered}
S_{i+1}=S_{i}+\left(A-S_{i} \frac{\beta_{1} I_{i}}{1+\alpha_{1} I_{i}}-S_{i} \frac{\beta_{2} D_{i}}{1+\alpha_{2} D_{i}}-\mu S_{i}-q S_{i}\right) \Delta t \\
+S_{i}\left(\sigma_{1} \xi_{i} \sqrt{\Delta t}+\frac{\sigma_{1}^{2}}{2}\left(\xi_{i}^{2}-1\right) \Delta t\right), \\
I_{i+1}=I_{i}+\left(S_{i} \frac{\beta_{1} I_{i}}{1+\alpha_{1} I_{i}}+S_{i} \frac{\beta_{2} D_{i}}{1+\alpha_{2} D_{i}}-(\rho+\mu+\delta) I_{i}\right) \Delta t \\
+I_{i}\left(\sigma_{2} \eta_{i} \sqrt{\Delta t}+\frac{\sigma_{2}^{2}}{2}\left(\eta_{i}^{2}-1\right) \Delta t\right), \\
D_{i+1}=D_{i}+\left((\mu+\delta) I_{i}-\gamma D_{i}\right) \Delta t+D_{i}\left(\sigma_{3} \zeta_{i} \sqrt{\Delta t}+\frac{\sigma_{2}^{2}}{2}\left(\zeta_{i}^{2}-1\right) \Delta t\right),
\end{gathered}
$$

where $\xi_{i}, \eta_{i}, \zeta_{i}(i=1,2, \ldots)$ are independent $\mathbf{N}$-distributed Gaussian random variables. 

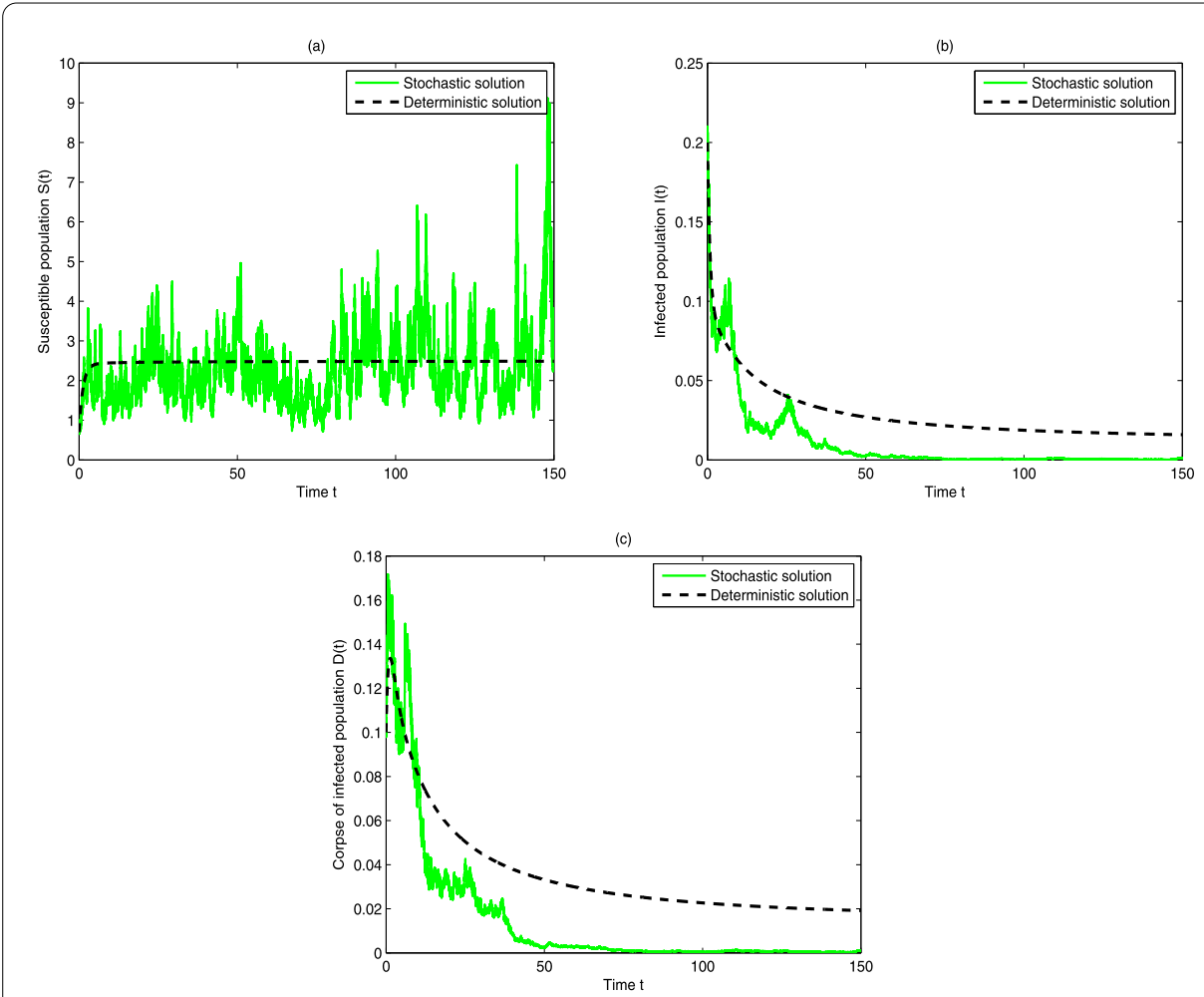

Figure 2 Extinction: the stochastic trajectory and deterministic trajectory of $S(t), /(t), D(t)$

Example 4.1 We take the parameters in system (1.3) as follows: $A=5, \alpha_{1}=2, \alpha_{2}=2$, $\beta_{1}=0.35, \beta_{2}=0.45, \mu=0.06, \delta=0.3, \rho=0.05, q=0.7, \gamma=0.5, \sigma_{1}=0.1, \sigma_{2}=0.1, \sigma_{3}=0.1$, and the initial value is $(S(0), I(0), D(0))=(0.7,0.2,0.1)$. We can obtain that

$$
\mathfrak{R}_{0}^{s}:=\frac{\beta_{1}}{\left(\mu+q+\frac{\sigma_{1}^{2}}{2}\right)\left(\rho+\mu+\delta+\frac{\sigma_{2}^{2}}{2}\right)}=1.1024>1 .
$$

Theorem 2.2 implies that system (1.3) has a unique stationary distribution(Fig. 1). From Fig. 1, we can see that the numbers of susceptible group, infected group, and infected corpse group are normally distributed.

Example 4.2 We take the parameters as follows: $A=2, \alpha_{1}=2, \alpha_{2}=2, \beta_{1}=0.05, \beta_{2}=0.2$, $\mu=0.1, \delta=0.5, \rho=0.1, q=0.7, \gamma=0.5, \sigma_{1}=0.5, \sigma_{2}=0.2, \sigma_{3}=0.2$, and the initial value is $(S(0), I(0), D(0))=(0.7,0.2,0.1)$. We can easily calculate the basic reproduction number $\Re_{0}=\frac{\beta_{1} A \gamma+\beta_{2} A(\mu+\delta)}{(\mu+q) \gamma(\rho+\mu+\delta)}=1.036>1$.

$$
\tilde{\Re}_{0}^{s}=\frac{\left(\beta_{1}+\beta_{2}\right) A}{(\mu+q)\left(\rho+\mu+\delta+\frac{\sigma_{2}^{2}}{2}\right)}=0.7576<1,
$$

which satisfies the condition of Theorem 3.1. So, the disease will go to extinction, that is to show that the white noise may lead to the extinction of disease. However, for deterministic models (1.2), the disease persists since $\Re_{0}>1$. Thus, the numerical simulations validate our theoretical results (Fig. 2). Figure 2 shows the change curves of the numbers of susceptible group, infected group, and infected corpse group over time. We can also see that 
the comparison of curves of the same variable makes the numerical simulation of model (1.3) closer to the reality.

\section{Conclusions}

In this paper, a new SIRD epidemic model of Ebola with double saturated incidence rates and vaccination is proposed and studied. Firstly, we construct suitable Lyapunov functions, we get that if $\Re_{0}^{s}=\frac{\beta_{1}}{\left(\mu+q+\frac{\sigma_{1}^{2}}{2}\right)\left(\rho+\mu+\delta+\frac{\sigma_{2}^{2}}{2}\right)}>1$, then system (1.3) has a unique stationary distribution. Next, we find that if $\tilde{\Re}_{0}^{s}=\frac{\left(\beta_{1}+\beta_{2}\right) A}{(\mu+q)\left(\rho+\mu+\delta+\frac{\sigma_{2}^{2}}{2}\right)}<1$, then the disease dies out with probability one. Finally, numerical simulations are shown to verify our results.

Some interesting topics deserve further consideration. On the one hand, one may propose some more realistic but complex models, such as considering the effects of impulsive perturbations on system (1.3). On the other hand, in our model (1.2), we only introduce the white noise into it, one can also introduce the colored noise into model (1.2). Moreover, our model is autonomous, it is interesting to investigate the nonautonomous system. We will leave these problems as our future work.

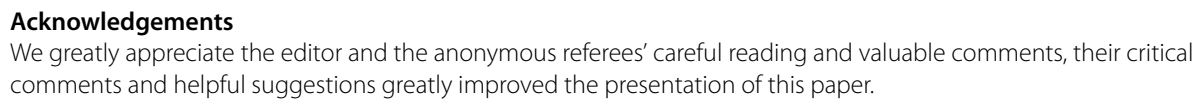

\section{Publisher's Note}

Springer Nature remains neutral with regard to jurisdictional claims in published maps and institutional affiliations.

Received: 28 June 2019 Accepted: 25 September 2019 Published online: 15 October 2019

References

1. Al-Darabsah, I., Yuan, Y.: A time-delayed epidemic model for Ebola disease transmission. Appl. Math. Comput. 290, 307-325 (2016)

2. Weitzand, J., Dushoff, J.: Modeling post-death transmission of Ebola: challenges for inference and opportunities for control. Sci. Rep. 5, 8751 (2015)

3. Towers, S., Patterson-Lomba, O., Castillo-Chavez, C.: Temporal variations in the effective reproduction number of the 2014 West Africa Ebola outbreak. PLOS Curr. Outbreaks (September 18, 2014)

4. Rachah, A., Torres, D.F.M.: Predicting and controlling the Ebola infection. Math. Methods Appl. Sci. Special Issue, 1-10 (2016)

5. Berge, T., Lubuma, J.M.S., Moremedic, G.M., et al.: A simple mathematical model for Ebola in Africa. J. Biol. Dyn. 11, 42-74 (2017)

6. Berge, T., Lubuma, J., Tass, A.J.O., et al.: Dynamics of host-reservoir transmission of Ebola with spillover potential to humans. Electron. J. Qual. Theory Differ. Equ. 2018, 14 (2018)

7. https://baike.so.com/doc/5512098-5747860.html

8. Driessche, P., Watmough, J.: Reproduction numbers and sub-threshold endemic equilibria for compartmental models of disease transmission. Math. Biosci. 180, 29-48 (2002)

9. Jiang, J.C., Shi, D.: Multigroup SIR epidemic model with stochastic perturbation. Physica A 390, 1747-1762 (2011)

10. Zhao, Y.N., Jiang, D.Q.: The extinction and persistence of the stochastic SIS epidemic model with vaccination. Physica A 392, 4916-4927 (2013)

11. Zhao, Y.N., Jiang, D.Q.: The threshold of a stochastic SIS epidemic model with vaccination. Appl. Math. Comput. 243, 718-727 (2014)

12. Teng, Z.D., Wang, L.: Persistence and extinction for a class of stochastic SIS epidemic models with nonlinear incidence rate. Physica A 451, 507-518 (2016)

13. Khan, T., Khan, A., Zaman, G.: The extinction and persistence of stochastic hepatitis B epidemic model. Chaos Solitons Fractals 108, 123-128 (2018) 
14. Djordjevic, J., Silva, C.J., Torres, D.F.M.: A stochastic SICA epidemic model for HIV transmission. Appl. Math. Lett. 84, $168-175(2018)$

15. Liu, Q., Jiang, D.Q., Shi, N.Z.: Threshold behavior in a stochastic SIQR epidemic model with standard incidence and regime switching. Appl. Math. Comput. 316, 310-325 (2018)

16. Lu, R.X., Wei, F.Y.: Persistence and extinction for an age-structured stochastic SVIR epidemic model with generalized nonlinear incidence rate. Physica A 513, 572-587 (2019)

17. Zhang, Y., Fan, K., Gao, S.J., et al.: Ergodic stationary of stochastic SIRS epidemic model incorporating media coverage and saturated incidence rate. Physica A 514, 671-685 (2019)

18. Zhang, X.B., Chang, S.C., Shi, Q.H., Huo, H.F.: Qualitative study of a stochastic SIS epidemic model with vertical transmission. Physica A 505, 805-817 (2018)

19. Khasminskii, R.: Stochastic Stability of Differential Equations, 2nd edn. Berlin, Heidelberg (2012)

20. Higham, D.: An algorithmic introduction to numerical simulation of stochastic differential equations. SIAM Rev. 43, 525-546 (2001)

Submit your manuscript to a SpringerOpen ${ }^{\circ}$ journal and benefit from:

- Convenient online submission

Rigorous peer review

Open access: articles freely available online

- High visibility within the field

- Retaining the copyright to your article

Submit your next manuscript at $\boldsymbol{\nabla}$ springeropen.com 